\title{
GRAPH COMPLEMENT CONJECTURE FOR CLASSES OF SHADOW GRAPHS
}

\author{
MONSIKARN JANSRANG* AND SIVARAM K. NARAYAN
}

Abstract. The real minimum semidefinite rank of a graph $G$, denoted $\mathrm{mr}_{+}^{\mathbb{R}}(G)$, is defined to be the minimum rank among all real symmetric positive semidefinite matrices whose zero/nonzero pattern corresponds to the graph $G$. The inequality $\operatorname{mr}_{+}^{\mathbb{R}}(G)+\operatorname{mr}_{+}^{\mathbb{R}}(\bar{G}) \leqslant|G|+2$ is called the graph complement conjecture, denoted $G C C_{+}$, where $\bar{G}$ is the complement of $G$ and $|G|$ is the number of vertices in $G$. A known definition of shadow graph $S(G)$ and a variant of this definition denoted $\operatorname{Shad}(G)$ are given. It is shown that $S(G)$ satisfies $G C C_{+}$when $G$ is a tree or a unicyclic graph or a complete graph. Under additional conditions on $\bar{G}$, it is shown that $S(G)$ satisfies $G C C_{+}$when $G$ is a $k$-tree or a chordal graph. Moreover, whenever $G$ satisfies $G C C_{+}$and $\bar{G}$ does not contain any isolated vertices, it is shown that $\operatorname{Shad}(G)$ satisfies $G C C_{+}$.

Mathematics subject classification (2020): 05C50, 15A03, 15A18, 15 B57.

Keywords and phrases: Shadow graphs, minimum semidefinite rank, graph complement conjecture.

\section{REFERENCES}

[1] Francesco Barioli, Wayne Barrett, Shaun M. Fallat, H. Tracy Hall, Leslie HogBEN, BRYAN SHADER, P. VAN DEN DRIESSCHE AND HEIN VAN DER HOlSt, Zero forcing parameters and minimum rank problems, Linear Algebra Appl., 433 (2): 401-411, 2010.

[2] Francesco Barioli, Wayne Barrett, Shaun M. Fallat, H. Tracy Hall, Leslie Hogben AND HEIN VAN DER HOLST, On the graph complement conjecture for minimum rank, Linear Algebra Appl., 436 (12): 4373-4391, 2012.

[3] Francesco Barioli, Shaun M. Fallat, Lon H. Mitchell and Sivaram K. Narayan, Minimum semidefinite rank of outerplanar graphs and the tree cover number, Electron. J. Linear Algebra, 22: 10-21, 2011.

[4] J. A. Bondy And U. S. R. MurTy, Graph theory, vol. 244 of Graduate Texts in Mathematics, Springer, New York, 2008.

[5] Matthew Booth, Philip Hackney, Benjamin Harris, Charles R. Johnson, Margaret Lay, Terry D. Lenker, Lon H. Mitchell, Sivaram K. Narayan, Amanda Pascoe and BRIAN D. SUTTON, On the minimum semidefinite rank of a simple graph, Linear Multilinear Algebra, 59 (5): 483-506, 2011.

[6] Matthew Booth, Philip Hackney, Benjamin Harris, Charles R. Johnson, Margaret Lay, Lon H. Mitchell, Sivaram K. Narayan, Amanda Pascoe, Kelly Steinmetz, Brian D. SUTTON AND WENDY WANG, On the minimum rank among positive semidefinite matrices with a given graph, SIAM J. Matrix Anal. Appl., 30 (2): 731-740, 2008.

[7] Andreas Brandstädt, VAn Bang Le And Jeremy P. SpinRad, Graph classes: a survey, SIAM Monographs on Discrete Mathematics and Applications, Society for Industrial and Applied Mathematics (SIAM), Philadelphia, PA, 1999.

[8] Richard Brualdi, Leslie Hogben and Bryan Shader, AiM Workshop Spectra of Families of Matrices described by Graphs, Digraphs, and Sign Patterns Final Report: Mathematical Results (revised), https://aimath.org/pastworkshops/matrixspectrum.html.07 2018

[9] G. Chartrand And P. Zhang, A First Course in Graph Theory, Dover books on Mathematics, Dover Publications, 2012. 
[10] Gary Chartrand, Linda Lesniak and Ping Zhang, Graphs \& Digraphs, Chapman \& Hall/CRC, 6th edition, 2015.

[11] Yves COLIN DE Verdière, Multiplicities of eigenvalues and tree-width of graphs, J. Combin. Theory Ser. B, 74 (2): 121-146, 1998.

[12] Jonathan L. Gross, Jay Yellen and Ping Zhang, Handbook of graph theory, Discrete Mathematics and its Applications (Boca Raton), CRC Press, Boca Raton, FL, second edition, 2014.

[13] AIM Minimum Rank-Special Graphs Work Group, Zero forcing sets and the minimum rank of graphs, Linear Algebra Appl., 428 (7): 1628-1648, 2008.

[14] Philip Hackney, Benjamin Harris, Margaret lay, Lon H. Mitchell, Sivaram K. NARAYAN AND AMANDA PASCOE, Linearly independent vertices and minimum semidefinite rank, Linear Algebra Appl., 431 (8): 1105-1115, 2009.

[15] Leslie Hogben, Orthogonal representations, minimum rank, and graph complements, Linear Algebra Appl., 428 (11-12): 2560-2568, 2008.

[16] Leslie Hogben, Handbook of linear algebra, Discrete Mathematics and its Applications (Boca Raton), CRC Press, Boca Raton, FL, second edition, 2014.

[17] Roger A. Horn and Charles R. Johnson, Matrix analysis, 2nd edition, Cambridge University Press, Cambridge, 2013.

[18] Russell MerRis, A survey of graph Laplacians, Linear and Multilinear Algebra, 39 (1-2): 19-31, 1995.

[19] Lon H. Mitchell, On the graph complement conjecture for minimum semidefinite rank, Linear Algebra Appl., 435 (6): 1311-1314, 2011.

[20] Lon H. Mitchell, Sivaram K. NARAyan And Andrew M. Zimmer, Lower bounds for minimum semidefinite rank from orthogonal removal and chordal supergraphs, Linear Algebra Appl., 436 (3): 525-536, 2012.

[21] John Sinkovic AND HeIn VAn DeR Holst, The minimum semidefinite rank of the complement of partial k-trees, Linear Algebra Appl., 434 (6): 1468-1474, 2011.

[22] HeIn VAN DER HOLST, Graphs whose positive semi-definite matrices have nullity at most two, Linear Algebra Appl., 375: 1-11, 2003.

[23] Douglas B. West, Introduction to graph theory, 2nd edition, Prentice Hall, Inc., Upper Saddle River, NJ, 2001. 